\title{
Análise de Desempenho de Códigos de Bloco com Proteção Desigual de Erros Utilizando Algoritmos de Decisão Suave
}

\author{
Rebecca C. de Albuquerque ${ }^{1}$, Daniel C. Cunha ${ }^{1}$ e Cecilio Pimentel ${ }^{2}$
}

\begin{abstract}
Resumo-O grau de proteção desigual de erros de um código linear para decodificação de Máxima Verossimilhança (MV) é medido pelo vetor de separação. Este trabalho avalia o desempenho, através de simulação computacional, de classes de proteção desigual de erros de um algoritmo de decisão suave sub-ótimo proposto por Chase. Comparações de desempenho entre o algoritmo de Chase e o algoritmo MV são apresentadas.

Palavras-Chave-Algoritmo de Chase, códigos de bloco, probabilidade de erro, proteção desigual de erros.

Abstract-The error protection level of a linear code for Maximum Likelihood decoding is measured by separation vector. This paper evaluate the performance, by computer simulations, of unequal error protection classes of a sub-optimal soft decision algorithm proposed by Chase. Perfomance comparisons between Chase algorithm and ML algorithm are presented.
\end{abstract}

Keywords - Chase algorithm, block codes, error probability, unequal error protection.

\section{INTRODUÇÃO}

A transmissão de sinais digitais, como por exemplo, imagem e vídeo, por meio da Internet e através das redes sem fio, fixas ou móveis, tem crescente importância prática. Uma técnica efetiva para esta transmissão consiste em oferecer níveis de proteção distintos a diferentes partes dos dados de informação. A esta técnica dá-se o nome de codificação com proteção desigual de erros (UEP, do inglês Unequal Error Protection) [1]. Para obter proteção desigual, os dados devem ser divididos em duas ou mais classes com diferentes prioridades. Com duas classes, por exemplo, os erros ocorridos na classe de mais alta prioridade são bastante prejudiciais à qualidade do sinal reconstruído e devem ser evitados ao máximo. Por outro lado, erros ocorridos na classe de prioridade mais baixa são mais toleráveis.

Este trabalho realiza uma avaliação de desempenho, através de simulações computacionais, de um algoritmo de decodificação por decisão suave (algoritmo de Chase [2]) para cada classe de proteção de um código de bloco UEP com transmissão BPSK em um canal com ruído aditivo Gaussiano Branco. O desempenho do algoritmo de Chase é comparado com o algoritmo de decodificação de Máxima Verossimilhança (MV) com o objetivo de mensurar a perda de desempenho de cada classe de proteção proporcionada por um algoritmo menos complexo. Na seção II, conceitos relativos à codificação

1 Núcleo de Pesquisa em Telecomunicações, POLI, Universidade de Pernambuco (UPE), Recife-PE, emails: rebeccacamile@gmail.com, dccunha@upe.poli.br.

2 Departamento de Eletrônica e Sistemas, Universidade Federal de Pernambuco (UFPE), Recife-PE, email:cecilio@ufpe.br.

Este trabalho recebeu suporte parcial do CNPq, no. 304173/2007-0.
UEP são descritos. Algumas propriedades do algoritmo de Chase são descritas na Seção III. Na Seção IV, resultados de simulação são apresentados. Por fim, a seção V, apresenta as conclusões e as perspectivas de trabalhos futuros.

\section{CodificaçÃo Com Proteção Desigual de Erros}

Considere um código linear binário $C(n, k)$ em que $n$ é o comprimento das palavras-código e $k$ é o tamanho do vetor de informação. A matriz geradora do código $C(n, k)$ é denotada por G. Assumindo que $w(\mathbf{u G})$ é o peso de Hamming da palavra-código $\mathbf{x}=\mathbf{u G}$ relativa ao vetor de informação $\mathbf{u}$, define-se o vetor de separação $\mathbf{S}=\left(S_{1}, \ldots, S_{k}\right)$ do código $C(n, k)$ de maneira que a $i$-ésima posição de $\mathbf{S}$ é dada por [1]

$$
S_{i}=\min \left\{w(\mathbf{u G}): \mathbf{u} \in G F(q)^{k}, u_{i} \neq 0\right\}, i=1, \cdots, k .
$$

O menor elemento de $\mathbf{S}$ é a distância mínima do código, denotada por $d$. Um código é dito ter proteção igual de erros (EEP, do inglês Equal Error Protection) se todos os elementos de $\mathbf{S}$ forem iguais, caso contrário, o código possui a propriedade UEP.

$\mathrm{O}$ vetor de separação $\mathbf{S}$ mede a proteção desigual de erros fornecida pelo código para decodificação MV. Sendo assim, a decodificação correta da $i$-ésima posição do vetor $\mathbf{u}$ ocorrerá caso menos de $\left\lfloor\frac{S_{i}-1}{2}\right\rfloor$ erros ocorram na palavra transmitida. Uma vez que cada posição do vetor de informação possui um nível de proteção individual, é possível agrupar as posições que possuem o mesmo nível de proteção em uma determinada classe de proteção. Para ilustrar os conceitos apresentados, considere o código de bloco linear $C(10,5)$, cuja matriz geradora é dada por

$$
\mathbf{G}=\left[\begin{array}{llllllllll}
1 & 0 & 0 & 0 & 0 & 1 & 1 & 1 & 1 & 0 \\
0 & 1 & 0 & 0 & 0 & 1 & 0 & 0 & 0 & 1 \\
0 & 0 & 1 & 0 & 0 & 1 & 1 & 0 & 0 & 1 \\
0 & 0 & 0 & 1 & 0 & 1 & 0 & 1 & 0 & 1 \\
0 & 0 & 0 & 0 & 1 & 1 & 0 & 0 & 1 & 1
\end{array}\right]
$$

e que possui vetor de separação $\mathbf{S}=(5,3,3,3,3)$. O primeiro bit de informação possui um nível de proteção $S_{1}=5$, enquanto os demais bits de informação possuem nível de proteção $S_{j}=3, j=2, \ldots, 5$. Desta maneira, podemos afirmar que $C(10,5)$ é um código UEP e possui duas classes de proteção distintas, denotadas por $\mathrm{Cp}_{1}$ (classe de maior proteção) e $\mathrm{cp}_{2}$ (classe de menor proteção).

\section{Algoritmo DE Chase}

O algoritmo de decisão suave considerado neste trabalho é o algoritmo de decodificação proposto por Chase [2]. 
Basicamente, o algoritmo de Chase necessita da sequência de valores reais observada na saída do canal, denotada por $\boldsymbol{\rho}=\left[\rho_{1}, \rho_{2}, \ldots, \rho_{n}\right]$, e da sequência binária gerada pelas decisões abruptas da sequência $\rho$. Os valores reais de $\rho$ representam o nível de confiabilidade referente a cada bit da palavra recebida. Quanto maior for o valor de confiabilidade, menor é a probabilidade de o bit correspondente ter sido afetado fortemente pelo ruído. Com isso, a decodificação de cada sequência $\rho$ depende da escolha do padrão de teste $\mathbf{T}$, vetor de $n$ bits com 1's nas posições menos confiáveis, que permite a obtenção do padrão de erro mais provável de ter ocorrido. A descrição completa do algoritmo de Chase pode ser encontrada em [2].

Existem três variantes do algoritmo de Chase baseadas no número de padrões $\mathbf{T}$ utilizados, determinado em função do parâmetro $p$. No algoritmo Chase-1, são gerados todos os padrões T com $\lfloor p / 2\rfloor$ 1's. No algoritmo Chase-2, são consideradas todas as combinações de 1 's nas $\left\lfloor\frac{p}{2}\right\rfloor$ posições menos confiáveis, portanto são produzidos $2^{\lfloor p / 2\rfloor}$ padrões $\mathbf{T}$. Por fim, existe ainda o algoritmo Chase-3, considerado o mais simples dos três, pois utiliza o menor número de padrões $\mathbf{T}$, $\left\lfloor\frac{p}{2}+1\right\rfloor$, com $m$ 1's nas $m$ posições menos confiáveis. De acordo com [2], é assumido em todas as variantes do algoritmo de Chase que $p=d$.

\section{Resultados de Simulações}

Dentre os três algoritmos de Chase existentes, foi escolhido o algoritmo Chase-2 por apresentar a melhor relação entre complexidade computacional e desempenho [2]. Portanto, foram realizadas simulações computacionais do algoritmo de decodificação MV e do algoritmo Chase-2 para o código de bloco $C(10,5)$ definido em (2). Para cada classe de proteção, foram extraídas curvas de probabilidade de erro de bit $\left(P_{b}\right)$ versus relação sinal-ruído $E_{b} / N_{0}$, em que $E_{b}$ é a energia por bit de informação e $N_{0}$ é a densidade espectral de potência do ruído Gaussiano.

As Figs. 1 e 2 ilustram o desempenho dos algoritmos MV e Chase-2 por classe de proteção para o código $C(10,5)$. O algoritmo Chase-2 foi implementado para $p=d$ (Fig. 1) e $p=5$ (Fig. 2), o que corresponde à geração de 2 e 4 padrões de teste $\mathbf{T}$, respectivamente. Cabe ressaltar que o segundo valor assumido para $p$ extrapola a concepção original do algoritmo Chase-2. Para uma $P_{b}=10^{-4}$, foram observadas perdas em $E_{b} / N_{0}$ ao compararmos o algoritmo Chase-2 com o algoritmo MV. Para a classe de proteção $\mathrm{Cp}_{1}$, o algoritmo Chase-2 $\operatorname{com} p=d$ ocasionou uma perda de aproximadamente $2 \mathrm{~dB}$, enquanto que para o algoritmo Chase- 2 com $p=5$, a perda foi de aproximadamente $1 \mathrm{~dB}$. Por outro lado, quando a classe de menor proteção $\left(\mathrm{Cp}_{2}\right)$ foi avaliada, as perdas obtidas foram menores. Para o algoritmo Chase-2 com $p=d$, a perda ocasionada foi de aproximadamente $0,5 \mathrm{~dB}$, enquanto que para o algoritmo Chase- 2 com $p=5$, a perda foi de aproximadamente $0,1 \mathrm{~dB}$. O desempenho da modulação BPSK não codificada também é mostrado nas figuras.

\section{CONClusões}

As curvas apresentadas neste trabalho revelam que o algoritmo Chase-2 implementado com $p=d$ não contempla uma separação pretendida para o desempenho das classes

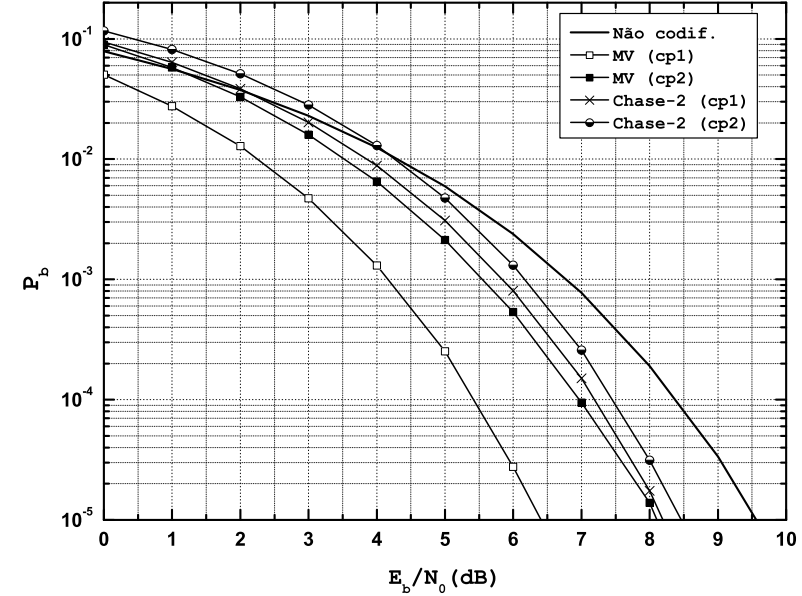

Fig. 1. Probabilidade de erro de bit versus relação sinal-ruído dos algoritmos MV e Chase-2 para o código $C(10,5)$ com modulação binária, canal com ruído aditivo Gaussiano Branco e $p=d$.

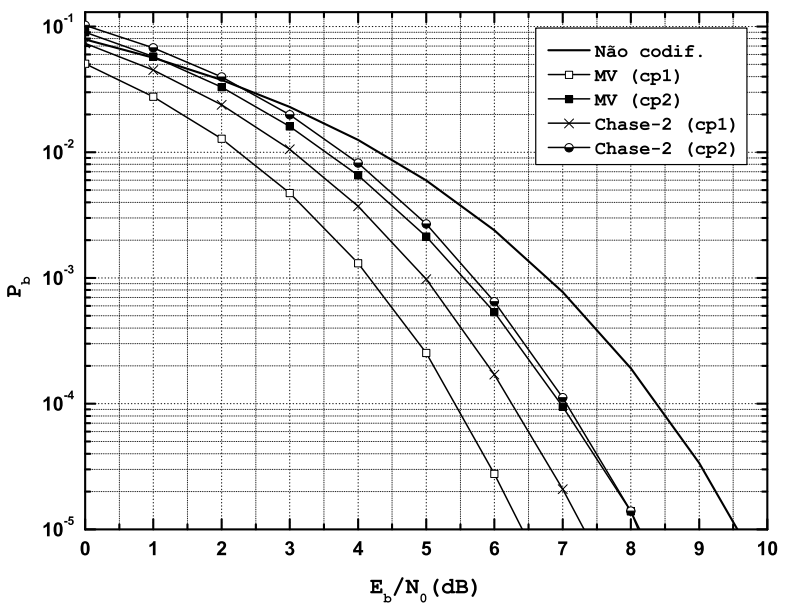

Fig. 2. Probabilidade de erro de bit versus relação sinal-ruído dos algoritmos MV e Chase-2 para o código $C(10,5)$ com modulação binária, canal com ruído aditivo Gaussiano Branco e $p=5$.

de proteção. A elevação do número de padrões de testes (usamos neste trabalho $p=5$ ) é eficaz para a classe de menor proteção em que os desempenhos dos algoritmos MV e Chase-2 tornam-se muito próximos, mas ainda existe uma lacuna importante entre o desempenho dos algoritmos na classe de maior proteção. Uma sugestão para trabalhos futuros é o projeto de um algoritmo de decisão suave para códigos UEP que tenha desempenho próximo do MV na classe de maior proteção. Os autores conjecturam que esta meta pode ser alcançada modificando-se o algoritmo de Chase para que este considere o vetor de separação no processo de decodificação.

\section{REFERÊNCIAS}

[1] B. Masnick e J. Wolf, "On Linear Unequal Error Protection Codes". IEEE Trans. on Information Theory, vol. IT - 3, n. 4, pp. 600-607, Out 1967.

[2] D. Chase, "A Class of Algorithms for Decoding Block Codes With Channel Measurement Information". IEEE Trans. on Information Theory, vol. IT - 18, n. 1, pp. 170-182, Jan 1972. 